\title{
Changes in von Willebrand factor and ADAMTS-13 in patients following arthroplasty
}

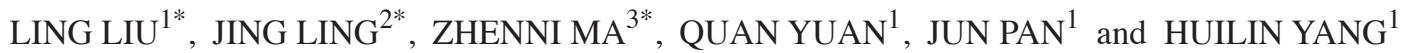 \\ ${ }^{1}$ Department of Orthopedics, Clinical Medical Research Center of Jiangsu Province, \\ The First Affiliated Hospital of Soochow University, Suzhou, Jiangsu 215006; ${ }^{2}$ Department of Hematology and Oncology, \\ Children's Hospital of Soochow University, Suzhou, Jiangsu 215003; \\ ${ }^{3}$ Key Laboratory of Thrombosis and Hemostasis of Ministry of Health, Jiangsu Institute of Hematology, \\ The First Affiliated Hospital of Soochow University, Suzhou, Jiangsu 215006, P.R. China
}

Received September 29, 2013; Accepted November 12, 2014

DOI: $10.3892 / \mathrm{mmr} .2014 .3041$

\begin{abstract}
The glycoprotein, von Willebrand factor (VWF) is a carrier protein for factor VIII. When bound to platelets and the extracellular matrix, it promotes aggregation or adhesion of platelets to areas of vascular damage. A disintegrin and metalloproteinase with thrombospondin motif, member 13 (ADAMTS13) cleaves between the tyr1605 and met1606 residues in the central A2 domain of VWF decreasing its activity. The levels of ADAMTS13 and VWF are positively correlated with the risk of developing thrombosis and inversely correlated with the risk of bleeding. A total of 93 patients were observed, who underwent total hip arthroplasty or total knee arthroplasty. Blood samples were collected preoperatively and on postoperative days (PODs) 1,2,3,5 and 7. Plasma levels of the ADAMTS13 antigen were determined using western blotting. The proteolytic activity was validated with the FRETS-VWF73 assay. VWF:Ag and VWF:RCo activity were measured using an enzyme-linked immunosorbent assay. Prothrombin time (PT), activated partial thromboplastin time (APTT), thrombin time (TT), antithrombin III and plasma fibrinogen levels were measured on a Sysmex ${ }^{\circledR}$ CA500 system with corresponding reagents. D-dimer levels were measured on a STA-R fully automated coagulation analyzer. The results demonstrated that, the levels of VWF antigen and activity in the patient increased from postoperative day (POD) 1. By contrast, the level of the ADAMTS13 antigen and its activity in the patients decreased
\end{abstract}

Correspondence to: Professor Huilin Yang, Department of Orthopedics, Clinical Medical Research Center of Jiangsu Province, The First Affiliated Hospital of Soochow University, 188 Shizi Street, Suzhou, Jiangsu 215006, P.R. China

E-mail: yanghuilin60@gmail.com

${ }^{*}$ Contributed equally

Key words: A disintegrin and metalloproteinase with thrombospondin motif, member 13, von Willebrand factor, total hip arthroplasty, total knee arthroplasty, venous thrombosis embolism significantly. Starting on POD1, fibrinogen and D-dimer levels increased. No significant changes were observed in PT, APTT and TT. It was concluded that the ADAMTS13 and VWF levels exhibited a marked association with thrombosis risk. The levels of ADAMTS13 and VWF may be potentially useful as markers for predicting thrombotic complications following arthroplasty and inhibiting the activity of VWF may be a novel prophylaxis to reduce postoperative deep venous thrombosis and pulmonary embolism.

\section{Introduction}

Hemostasis is a physiological host defense mechanism focused on the arrest of bleeding following vascular injury. It preserves vascular integrity and prevents excessive blood loss. Arrest of bleeding requires rapid formation of hemostatic plugs at sites of vascular injury to prevent exsanguination. This depends on a complex series of regulatory pathways that activate the platelets and the coagulation system. By contrast, excessive activation of the coagulation system may lead to thrombosis (1).

Total hip arthroplasty (THA) and total knee arthroplasty (TKA) have been performed with increasing frequency in previous years. In patients undergoing THA or TKA, different patterns of altered venous hemodynamics and hypercoagulability have been identified, thus the rate of distal deep venous thrombosis (DVT) has increased (2). In addition, the risk of venous thrombosis embolism (VTE) in THA and TKA is among the highest for all surgical procedures (3). VTE can be treated, however when left untreated it may be fatal (4).

Thrombosis and hemostasis are dependent upon the involvement of von Willebrand factor (VWF) and the involvement of VWF is a result of competition between the biosynthesis of large VWF multimers and their degradation by A disintegrin and metalloproteinase with thrombospondin motif, member 13 (ADAMTS13) (5). ADAMTS13 is a member of a family of proteases termed ADAMTSs (6,7). ADAMTS13 has been identified as a VWF cleaving protease, which cleaves between the Tyr1605 and Met1606 residues in the central A2 domain of VWF (8-10) to inhibit the activity of VWF. VWF is a carrier protein for factor VIII (FVIII) and once bound to platelets and the extracellular matrix may promote platelet aggregation 
or platelet adhesion to areas of vascular damage (5). Several studies have found that FVIII and VWF levels were independently associated with venous thromboembolism and suggested that VWF may make a significant contribution to the risk of venous thrombosis, independent of FVIII $(11,12)$.

In the present study, blood samples were collected from patients who underwent a THA or TKA and the changes of procoagulant/fibrinolytic factors were analyzed, particularly VWF and ADAMTS13.

\section{Patients and methods}

Patients. This study conformed to the ethical guidelines of the 2004 Declaration of Helsinki and was approved by the institutional Ethics Committee at the First Affiliated Hospital of Soochow University, (Suzhou, China). Between June 2012 and March 2013, a total of 93 patients (58 females and 35 males; age, 44-84 years; mean age, 59.5 years) who underwent a THA or TKA were enrolled in the present study after written informed consent had been provided. The exclusion criterion was a history of abnormal bleeding.

Collection and processing of blood samples. Blood samples were obtained on the day of surgery and the mornings of the first, second, third, fifth and seventh postoperative days (PODs). The blood samples were collected by venipuncture into plastic tubes containing 1/9th volume $0.109 \mathrm{~mol} / \mathrm{l}$ buffered trisodium citrate. For the analysis of prothrombin time (PT; \%), activated partial thromboplastin time (APTT), thrombin time (TT), fibrinogen level, ADAMTS13 level and VWF level, platelet poor plasma was obtained by centrifugation at 2,500 $\mathrm{x} g$ for $15 \mathrm{~min}$ at $4^{\circ} \mathrm{C}$ and stored at $-80^{\circ} \mathrm{C}$ until further analysis. The normal human plasma pool was composed of the plasma of 25 normal individuals with different ABO blood groups.

Coagulation assessment. PT, APTT, TT, antithrombin III and plasma fibrinogen were measured on a Sysmex ${ }^{\circledR}$ CA500 system (Sysmex Corporation, Chuo-ku, Japan). PT was measured using the Thrombore ${ }^{\circledR} \mathrm{S}$ reagent (Siemens Healthcare Diagnostics, Marburg, Germany), PT international normalized ratio was calculated as the reaction time of the sample (sec) divided by the reaction time of the normal plasma (sec). APTT was measured using the Dade ${ }^{\circledR}$ Actin ${ }^{\circledR}$ Activated Cephaloplastin reagent (Siemens Healthcare Diagnostics). TT was measured using the Test Thrombin reagent (Siemens Healthcare Diagnostics). Antithrombin III was measured using the Berchrom ${ }^{\circledR}$ Antitrombina III reagent (Siemens Healthcare Diagnostics). D-dimer was measured on a STA-R fully automated coagulation analyzer with the corresponding reagents (Diagnostica Stago, Asnieres, France).

Plasma ADAMTS13 antigen determination. Plasma ADAMTS13 antigen was determined by western blotting using known concentrations of a recombinant ADAMTS13, which was provided by Jiangsu Institute of Hematology (Jiangsu, China), as a reference (13). Each sample was subjected to an $8 \%$ SDS-PAGE together with a serial dilution of recombinant ADAMTS13 (from 0.5-2 $\mu \mathrm{M}$ ) and then analyzed by western blotting with a 1:3,000 dilution of anti-human ADAMTS13 rabbit polyclonal antibody (ab28274; Abcam, Cambridge,
UK) in $0.1 \%$ Tween-20 in phosphate-buffered saline (PBS). Chemiluminescence was detected with SuperSignal West Pico (Thermo Scientific, Rockford, IL, USA). Proteins were quantified with the calibration curve of recombinant ADAMTS13, determined by densitometry with ImageJ software $1.46 \mathrm{r}$ (National Institutes of Health, Bethesda, MA, USA).

Cleavage of fluorescence resonance energy transfer substrate VWF73 by plasma ADAMTS13. Fluorescence resonance energy transfer substrate VWF73 (FRETS-VWF73) was provided by Dr Xinglong Zheng (The Children's Hospital of Philadelphia, Philadelphia, PA, USA). FRETS-VWF73 is a chemically synthesized fluorogenic peptide, containing the 73 -amino-acid residues between D1596 and R1668 in the A2 domain of VWF (14). The normal human plasma (NHP) from 25 normal individuals with different ABO blood groups was mixed as the standard plasma. The blood samples were diluted in a pH 6.0 buffer containing $5 \mathrm{mmol} / 1$ Bis-Tris, $25 \mathrm{mmol} / 1 \mathrm{CaCl}_{2}$ and $0.005 \%$ Tween-20. The NHP was diluted at the ratio of $1: 12.5,1: 25,1: 50,1: 100,1: 200$ and 1:400, as standard. A total of $50 \mu \mathrm{l}$ of the diluted samples was added to an enzyme immunoassay/radioimmunoassay plate (Costar, Corning, NY, USA) and then $50 \mu \mathrm{l}$ of fluorescein-labeled VWF73 was added to each well as the substrate and mixed well. After $5 \mathrm{~min}$, the absorbance of each sample was read at 485/530 nm every 2 min 30 times with a Varioskan ${ }^{\mathrm{TM}}$ Flash Multimode Reader (Thermo Fisher Scientific Inc., Vantaa, Finland)

VWF:Ag enzyme-linked immunosorbent assay (ELISA). VWF:Ag was measured by an ELISA with paired antibodies against human VWF, SZ29 and SZ34 from Jiangsu Institute of Hematology (Suzhou, China) (15). Microtiter plates (96 well) were coated overnight at $4^{\circ} \mathrm{C}$ with $100 \mu \mathrm{l} /$ well of monoclonal anti-VWF antibody SZ-29 (1:1,000; Jiangsu Institute of Hematology) (15) in coating buffer (0.05 M carbonate/bicarbonate buffer, $\mathrm{pH}$ 9.6). The plates were washed three times with $0.1 \%$ Tween-20 in PBS (PBST) and blocked overnight at $4^{\circ} \mathrm{C}$ with $2 \%$ (w/v) bovine serum albumin (BSA) in PBST, followed by washing with PBST. Standard plasma pool dilutions (1:20, 1:50, 1:100, 1:200, 1:500 and 1:1,000) and diluted test samples, all in $0.2 \%$ BSA-PBST, were added in duplicate to the wells and incubated for $2 \mathrm{~h}$ at $37^{\circ} \mathrm{C}$. Following washing, the samples were incubated with an anti-human VWF mouse monoclonal antibody SZ-34 labeled with horseradish peroxidase (HRP; 1:6,000 in 0.2\% BSA-PBST) obtained from the Jiangsu Institute of Hematology (15) for $2 \mathrm{~h}$ at $37^{\circ} \mathrm{C}$ and then detected by tetramethyl benzidine (TMB; Thermo Scientific) reaction and absorbance measurement as described previously (16).

VWF: RCo-ELISA. Microtiter wells (Nunc-immunoplate; Nunc AS, Kamstrup, Denmark) were coated with $100 \mu \mathrm{l}$ of $\mathrm{mAb} \mathrm{SZ}-151$ at $10 \mu \mathrm{g} / \mathrm{ml}$ in coating buffer and incubated overnight at $4^{\circ} \mathrm{C}$. The wells were washed with PBST three times and then blocked with $2 \%$ BSA-PBST for $2 \mathrm{~h}$ at $37^{\circ} \mathrm{C}$. Subsequently, the wells were incubated with TBST $(0.025 \mathrm{M}$ Tris-buffered saline with $0.1 \%$ Tween-20, pH 7.4) containing $4 \mu \mathrm{g} / \mathrm{ml}$ recombinant fragment of platelet glycoprotein Ib $\alpha$ (rfGPIb $\alpha$ ) for $2 \mathrm{~h}$ at $37^{\circ} \mathrm{C}$. Following washing the plates six times with TBST, the mixed-plasma pool as standard (diluted 
A

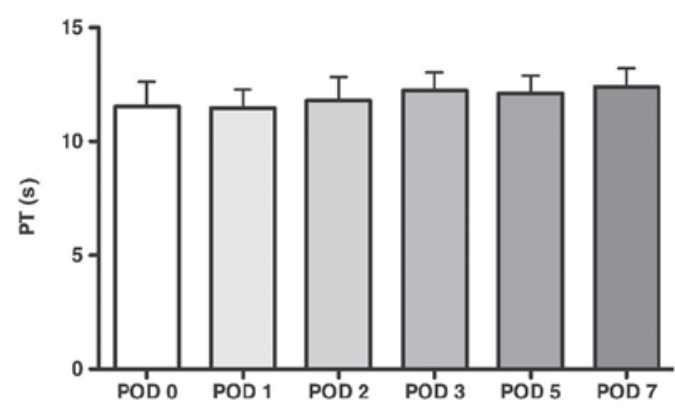

C

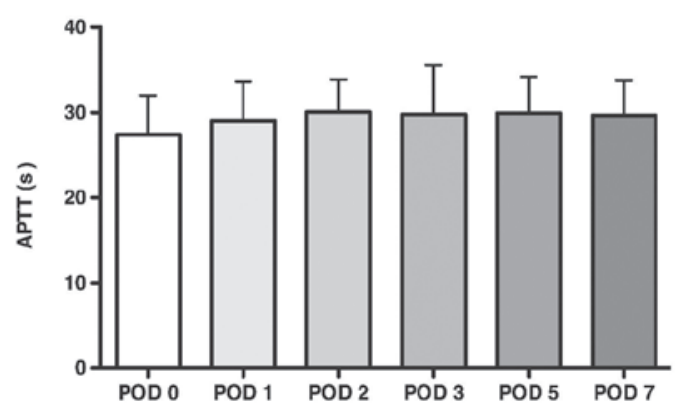

B

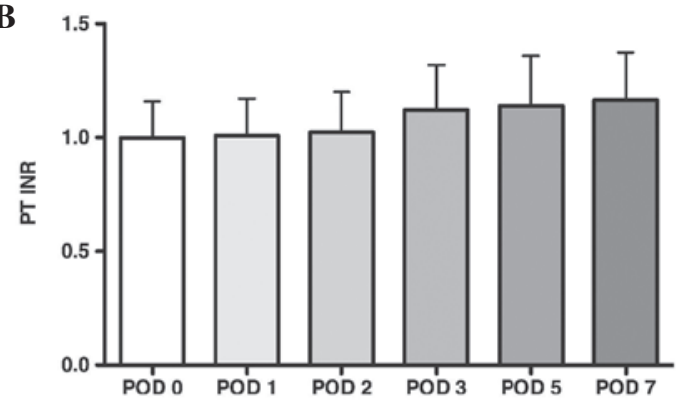

D

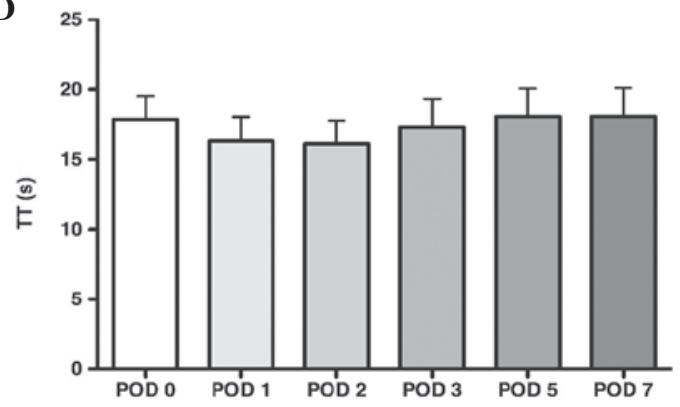

Figure 1.PT, APTT and TT levels. (A) PT level. (B) The PT-INR was obtained by conversion of the prothrombin ratio into internationally comparable values using the International Sensitivity Index. The prothrombin international normalized ratio was obtained by divinding the reaction time of the sample by the reaction time of the normal plasma pool. (C) APTT level. (D) TT level. (A), (C) and (D) were measured on a Sysmex ${ }^{\circledR}$ CA500 system (Sysmex $^{\circledR}$ Corporation, Chuo-ku, Japan). The mean PT, APTT and TT levels remained within reference limits throughout the entire observation period. The results are expressed as the mean \pm standard deviation; "P<0.05, compared with POD0. PT, prothrombin time; APTT, activated partial thromboplastin time; TT, thrombin time; POD, post-operative day; INR, international normalized ratio.

from 1:50 to 1:3,200) and test samples (diluted 1:50) containing $760 \mu \mathrm{g} / \mathrm{ml}$ ristocetin (Sigma, St. Louis, MO, USA) were added to the plates with captured rfGPIbo and incubated for $2 \mathrm{~h}$ at $37^{\circ} \mathrm{C}$. Following washing, bound VWF was incubated with HRP-conjugated rabbit anti-human VWF antibody (P0226; Dako-Cytomation, Glostrup, Denmark) for $1 \mathrm{~h}$ at $37^{\circ} \mathrm{C}$ and was detected with TMB. The reaction was stopped with $3 \mathrm{M}$ $\mathrm{H}_{2} \mathrm{SO}_{4}$ and the absorbance of each sample was read at $450 \mathrm{~nm}$, as previously described (16).

Statistical analysis. All results are expressed as the mean \pm standard deviation. Statistical significance was analyzed using the Friedman's test (a repeated measures analysis of variance for nonparametric data). $\mathrm{P}<0.05$ was considered to indicate a statistically significant difference. Statistical calculations were performed using Prism5 (GraphPad Software, La Jolla, CA, USA).

\section{Results}

Patient complications. Of the 97 patients primarily enrolled in the present study, four were excluded from the analysis due to the requirement for an intraoperative transfusion of fresh frozen plasma. A total of 51 patients underwent THA and 42 had a TKA. During the 7 day postoperative observation period, no apparent clinical thromboembolic complications were recorded. The demographic data are summarized in Table I.
Table I. Demographic data.

\begin{tabular}{lc}
\hline Characteristic & Variable \\
\hline Female & $58(62.36 \%)$ \\
Male & $35(37.63 \%)$ \\
Age (years) & $59.5(44-84)$ \\
Total hip arthroplasty & $51(54.83 \%)$ \\
Total knee arthroplasty & $42(45.16 \%)$ \\
\hline
\end{tabular}

Total number of patients $=93$. Values are presented as a number (percentage of patients) or median.

PT, APTT and TT levels. PT, APTT and TT were measured on a Sysmex CA500 system. The reference ranges were: PT, 10.8-13.6 sec; PT-INR, 0.800-1.500 sec; APTT, 23.0-37.0 sec; and TT, 14.0-21.0 sec, respectively. The results of the PT, APTT and TT are presented in Fig. 1. The mean PT, APTT and TT remained within reference limits throughout the entire observation period.

Antithrombin III, plasma fibrinogen and D-dimer levels. Antithrombin III and plasma fibrinogen were measured with a Sysmex CA500 system. D-dimer was measured on a STA-R fully automated coagulation analyzer. The reference ranges are as follows: Antithrombin III, 70-125\%; fibrinogen, 
$\mathbf{A}$

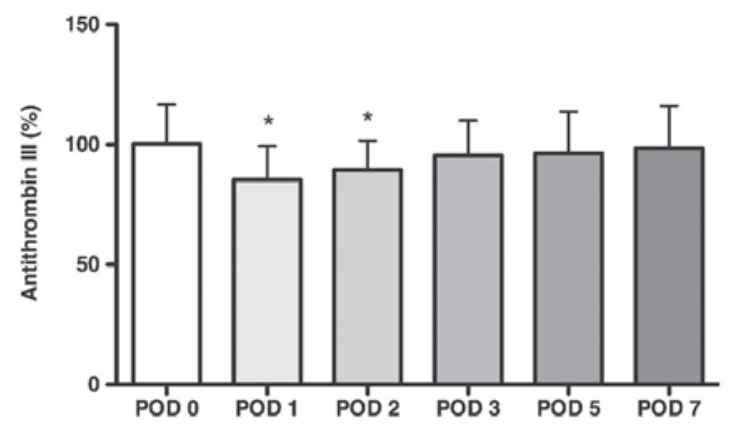

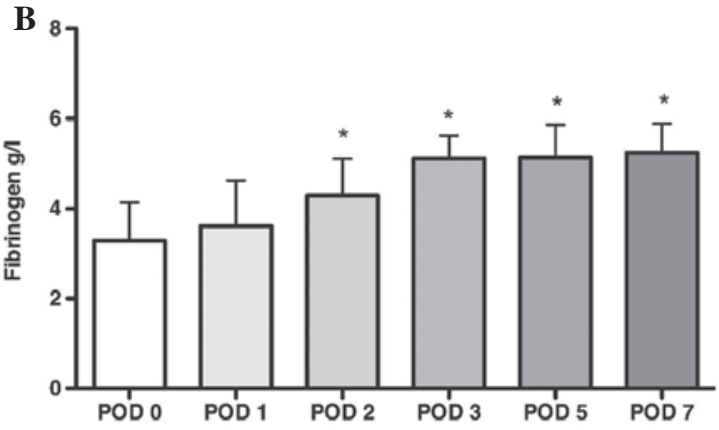

C

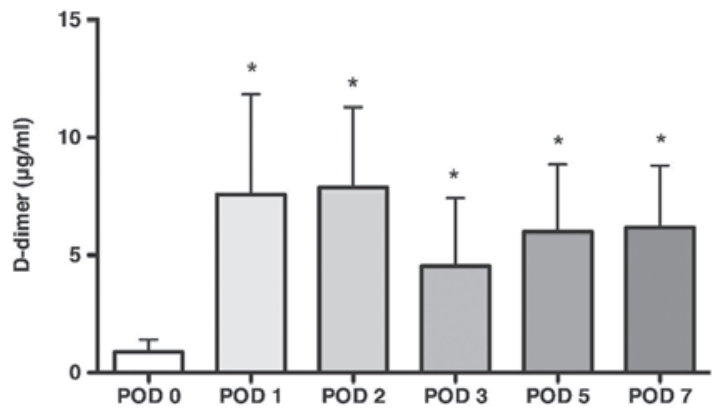

Figure 2. Antithrombin III, plasma fibrinogen and D-dimer levels. (A) Antithrombin III and (B) plasma fibrinogen levels were measured with a Sysmex ${ }^{\circledR}$ CA500 system (Sysmex ${ }^{\circledR}$ Corporation, Chuo-ku, Japan). (C) D-dimer levels were measured on a STA-R fully automated coagulation analyzer. The mean level of antithrombin III was found to be significantly below preoperative values on POD1 and POD2; however, it remained within the normal range and returned to preoperative values by POD3. On POD1, D-dimer levels were observed to increase significantly above preoperative levels. From POD2, the mean level of fibrinogen also increased significantly. During the 7 days following surgery, D-dimer and fibrinogen continued to increase. The results are expressed as the mean \pm standard deviation; ${ }^{*} \mathrm{P}<0.05$, compared with POD0. POD, post-operative day.

A

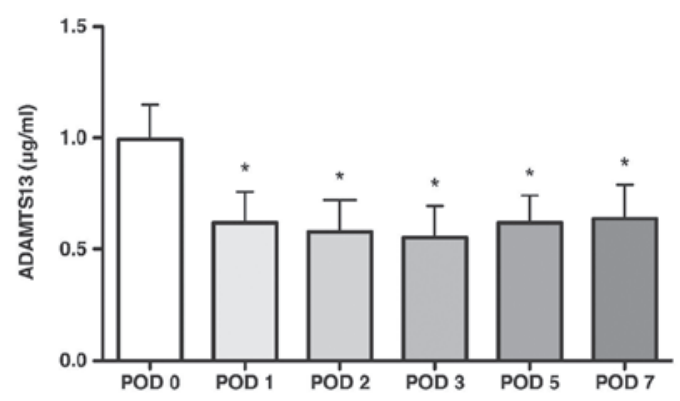

C

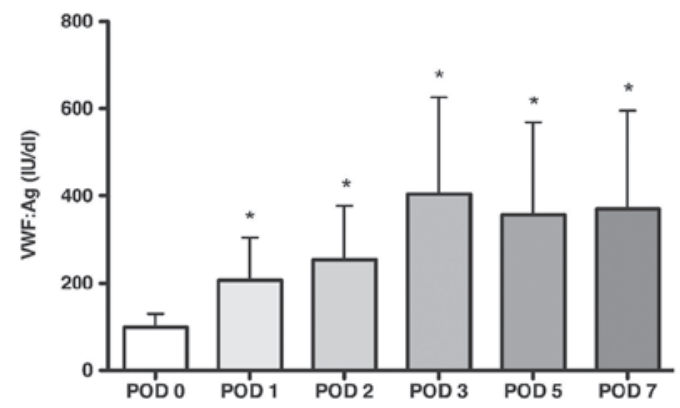

B

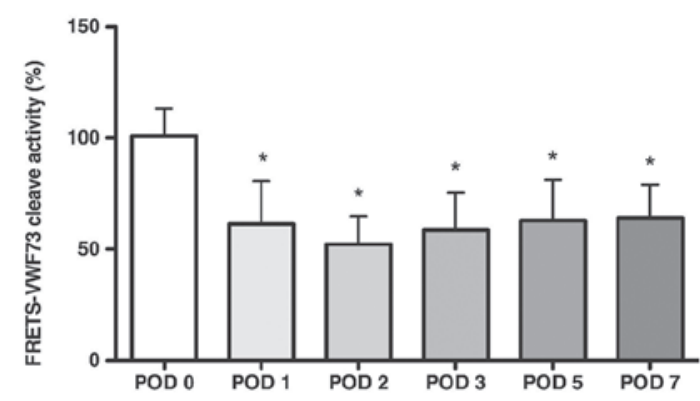

D

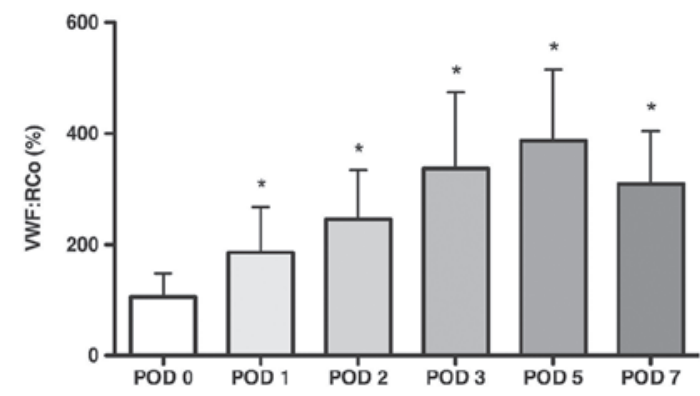

Figure 3. ADAMTS13 and VWF levels. (A) To examine the concentration of plasma ADAMTS13, equivalent fractions of each sample were subjected to $8 \%$ SDS-PAGE followed by western blot analysis. The result indicates that the mean level of plasma ADAMTS13 decreased continuously from POD1 compared with the preoperative level. (B) FRETS-VWF73 assay was used to validate the proteolytic activity of the ADAMTS13. The plasma ADAMTS13 activity was defined as the value divided by that of the normal human plasma diluted at 1:25 and multiplied by 100. The mean level of plasma ADAMTS13 proteolytic activity was also decreased from POD1. (C) VWF antigen and (D) VWF activity levels were measured with ELISA as described previously (14). The present results revealed that the VWF antigen and activity were increased significantly above preoperative levels as the plasma ADAMTS13 and its proteolytic activity decreased. The results are expressed as the mean \pm standard deviation; " $\mathrm{P}<0.05$, compared with POD0. VWF, von Willebrand factor; ADAMTS, a disintegrin and metalloproteinase with thrombospondin motifs; POD, post-operative day. 
2.000-5.000 g/l; and D-dimer, 0.01-0.5 $\mu \mathrm{g} / \mathrm{ml}$. The results for antithrombin III, plasma fibrinogen and D-dimer are presented in Fig. 2. The mean level of antithrombin III was observed to be significantly below preoperative values on POD1 and POD2 $(\mathrm{P}<0.05)$, however it remained within the normal range and returned to preoperative values by POD3. On POD1, D-dimer was observed to increase significantly above preoperative levels. From POD2, the mean level of fibrinogen also increased significantly. During POD7 D-dimer and fibrinogen levels remained raised $(\mathrm{P}<0.05)$.

ADAMTS13 and VWF. The results of the ADAMTS13 and VWF are presented in Fig. 3. To examine the concentration of plasma ADAMTS13, equivalent fractions of each sample were subjected to an $8 \%$ SDS-PAGE followed by western blot analysis. The result indicates that the mean level of plasma ADAMTS13 decreased continuously from POD1 compared with the preoperative level $(\mathrm{P}<0.05)$. The FRETS-VWF73 assay was used to validate the proteolytic activity of the ADAMTS13. VWF73 is a peptide fragment from the VWF A2 domain that contains the proteolytic site of ADAMTS13. The plasma ADAMTS13 activity was defined as the value divided by that of the normal human plasma diluted at 1:25 and multiplied by 100 . The mean level of plasma ADAMTS13 proteolytic activity also decreased from POD1 $(\mathrm{P}<0.05)$. VWF antigen and activity were measured with ELISA as described previously (16). The present results demonstrated that the VWF antigen and activity were increased significantly above preoperative levels $(\mathrm{P}<0.05)$ as the plasma ADAMTS13 and its proteolytic activity decreased.

\section{Discussion}

A total of 93 patients were assessed, who underwent either a THA or a TKA The present study demonstrated that no significant changes were observed for PT, APTT and TT; the mean level of antithrombin III was found to be significantly below preoperative values on POD1 and POD2 and the fibrinogen and D-dimer levels were increased continuously during the 7 days following surgery. The levels of VWF antigen and activity were increased significantly above preoperative levels, and the plasma ADAMTS13 and its proteolytic activity decreased continuously from POD1 to POD7.

A study of 252 patients who underwent a total joint arthroplasty revealed that the prevalence of DVT was $32 \%(16 \%$ distal, 16\% proximal) following THA and 66\% (50\% distal, $16 \%$ proximal) following TKA, without routine chemical or mechanical prophylaxis (17). A prospective epidemiological study was performed in 19 centers across Asia, in patients undergoing elective THA, TKA or hip fracture surgery (HFS) without pharmacological thromboprophylaxis. The results revealed that total DVT and proximal DVT rates were highest in TKA patients (58.1 and $17.1 \%$, respectively), followed by HFS patients (42.0 and 7.2\%, respectively) and THA patients (25.6 and $5.8 \%$, respectively). DVT was more frequent in female patients aged at least 65 years. The rate of venographic thrombosis in the absence of thromboprophylaxis following major joint surgery in Asian patients is similar to that previously reported in patients in Western countries (18).

There is evidence that, during surgery, dysfunction of the coagulation activation system may be responsible for hemor- rhage. In addition, anesthetic techniques have also been observed to affect hemorheological and hemostasiological parameters (19). These clinically significant hemorheological and hemostasiological alterations include: Hyperreagibility of platelets with increased aggregation and adhesion tendency; changes in fibrinogen, albumin and globulin concentrations, which affect viscosity and erythrocyte aggregation and impairment of deformability; increase in clotting factors and disturbance of fibrinolysis (20-22). Patients undergoing surgical procedures have been subject to investigation, in which thrombin activation parameters, clotting factors, fibrinolysis and thrombelastographic changes were examined in order to determine whether hypercoagulation occurs (23-27). The present study revealed that no significant changes were observed for PT, APTT and TT; the mean level of antithrombin III was found to be significantly below preoperative values on POD1 and POD2, and the fibrinogen and D-dimer levels were increased continuously during the 7 days following surgery. These results are consistent with previous studies $(23,27)$. It was also observed that the levels of VWF antigen and activity were increased significantly above preoperative levels as the plasma ADAMTS13 and its proteolytic activity decreased continuously from POD1 to POD7. By contrast, following surgery, a number of secretagogues, including thrombin, fibrin, histamine, the C5b-9 complement complex and several inflammatory cytokines triggered the secretion of VWF from its storage site in the Weibel-Palade body. While constitutively secreted multimers of VWF are of a relatively small size, the multimers stored within the Weibel-Palade body are the largest, most biologically potent form (28). By contrast, as the proteolytic activity of ADAMTS13 was impaired, the feedback proteolysis of VWF by ADAMTS-13 was decreased. The ultra large VWF in the plasma, which was secreted from the Weibel-Palade body, and its activity increased.

A population-based patient-control study was undertaken to elucidate the roles of the ABO blood group, VWF and clotting factor VIII in the process of DVT (11). Using univariate analysis, it was identified that blood group, VWF concentration and factor VIII concentration were all associated with DVT. The present study also suggested that the thrombosis risk associated with high VWF was mediated through factor VIII (11). Another study with samples obtained from 19,237 adults demonstrated that Factor VIII and VWF were linearly associated with increased risk of venous thromboembolism and suggested that VWF may make a significant contribution to the risk of venous thrombosis, independent of FVIII (12). VTE is a serious complication following major orthopedic surgery and $\sim 10 \%$ of mortality in hospitals is attributed to PE. There are several strategies to prevent this, including pharmacological and mechanical approaches (29). As high VWF is an independent risk factor of venous thrombosis, inhibiting the activity of VWF may be a novel form of prophylaxis to reduce postoperative DVT and PE, such as a monoclonal antibody SZ123, which was demonstrated to prevent in vivo thrombus formation by inhibiting VWF A3-collagen and VWF A1-platelet interactions (30).

In conclusion, the plasma ADAMTS13 and its proteolytic activity decreased as the level of VWF antigen and activity were increased significantly above preoperative levels 
following surgery. It is expected that the ADAMTS13 and VWF levels may be potentially useful markers in predicting thrombotic complications following arthroplasty and inhibiting the activity of VWF may be a novel prophylaxis to reduce postoperative DVT and PE.

\section{Acknowledgements}

The authors would like to thank Dr X. Long Zheng for providing FRETS-VWF73. The abstract of the present study was presented at the $34^{\text {th }}$ SICOT Orthopaedic World Congress, Hyderabad, India, October 17-19, 2013. The present study was supported by the Natural Science Foundation of Jiangsu Province (no. BK20140285) and the Jiangsu Provincial Special Program of Medical Science (no. BL2012004).

\section{References}

1. Anderson JA, Lim W and Weitz JI: Genetics of coagulation: what the cardiologist needs to know. Can J Cardiol 29: 75-88, 2013.

2. Fisher WD: Impact of venous thromboembolism on clinical management and therapy after hip and knee arthroplasty. Can J Surg 54: 344-351, 2011.

3. Falck-Ytter Y,Francis CW, Johanson NA, et al; American College of Chest Physicians: Prevention of VTE in orthopedic surgery patients: Antithrombotic Therapy and Prevention of Thrombosis, 9th ed: American College of Chest Physicians Evidence-Based Clinical Practice Guidelines. Chest 141 (2 Suppl): e278S-e325S, 2012.

4. Barrack RL: Current guidelines for total joint VTE prophylaxis: dawn of a new day. J Bone Joint Surg Br 94: 3-7, 2012.

5. Sadler JE: von Willebrand factor: two sides of a coin. J Thromb Haemost 3: 1702-1709, 2005.

6. Zheng X, Chung D, Takayama TK, Majerus EM, Sadler JE and Fujikawa K: Structure of von Willebrand factor-cleaving protease (ADAMTS13), a metalloprotease involved in thrombotic thrombocytopenic purpura. J Biol Chem 276: 41059-41063, 2001.

7. Porter S, Clark IM, Kevorkian L and Edwards DR: The ADAMTS metalloproteinases. Biochem J 386: 15-27, 2005.

8. Tsai HM: Physiologic cleavage of von Willebrand factor by a plasma protease is dependent on its conformation and requires calcium ion. Blood 87: 4235-4244, 1996.

9. Furlan M, Robles R and Lämmle B: Partial purification and characterization of a protease from human plasma cleaving von Willebrand factor to fragments produced by in vivo proteolysis. Blood 87: 4223-4234, 1996.

10. Fujikawa K, Suzuki H, McMullen B and Chung D: Purification of human von Willebrand factor-cleaving protease and its identification as a new member of the metalloproteinase family. Blood 98: 1662-1666, 2001.

11. KosterT,Blann AD,BrietE, Vandenbroucke JP and RosendaalFR: Role of clotting factor VIII in effect of von Willebrand factor on occurrence of deep-vein thrombosis. Lancet 345: 152-155, 1995.

12. Tsai AW, Cushman M, Rosamond WD, et al: Coagulation factors, inflammation markers, and venous thromboembolism: the longitudinal investigation of thromboembolism etiology (LITE). Am J Med 113: 636-642, 2002.
13. Wang AY, Liu F, Ma ZN, Dong NZ, Zhang JY and Ruan CG: Research on the C-terminal domain of ADAMTS13 regulates its cleaving activity. Zhonghua Xue Ye Xue Za Zhi 31: 830-834, 2010 (In Chinese).

14. Kokame K, Nobe Y, Kokubo Y, Okayama A and Miyata T: FRETS-VWF73, a first fluorogenic substrate for ADAMTS13 assay. Br J Haematol 129: 93-100, 2005.

15. Ruan CG, Xi XD and Gu JM: Studies on monoclonal antibodies to human von Willebrand factor. Zhonghua Nei Ke Za Zhi 25: 547-550, 1986 (In Chinese).

16. Zhao Y, Gu Y, Ji S, Yang J, Yu Z and Ruan C: Development of an ELISA method for testing VWF ristocetin cofactor activity with improved sensitivity and reliability in the diagnosis of von Willebrand disease. Eur J Haematol 88: 439-445, 2012.

17. Clarke MT, Green JS, Harper WM and Gregg PJ: Screening for deep-venous thrombosis after hip and knee replacement without prophylaxis. J Bone Joint Surg Br 79: 787-791, 1997.

18. Piovella F, Wang CJ, Lu H, et al: Deep-vein thrombosis rates after major orthopedic surgery in Asia. An epidemiological study based on postoperative screening with centrally adjudicated bilateral venography. J Thromb Haemost 3: 2664-2670, 2005.

19. Spirin VA, Khomenko NM and Vasil'kov VG: Hemostasis system in patients with bile duct diseases undergoing surgery under peridural anesthesia and neuroleptanalgesia. Anesteziol Reanimatol: 55-57, 1982 (In Russian).

20. Bergqvist D: Assessment of the risk and the prophylaxis of venous thromboembolism in surgical patients. Pathophysiol Haemost Thromb 33: 358-361, 2003.

21. Müller R and Musikić P: Hemorheology in surgery - a review. Angiology 38: 581-592, 1987.

22. Picker SM: In-vitro assessment of platelet function. Transfus Apher Sci 44: 305-319, 2011.

23. Lindberg F, Rasmussen I, Siegbahn A and Bergqvist D: Coagulation activation after laparoscopic cholecystectomy in spite of thromboembolism prophylaxis. Surg Endosc 14: 858-861, 2000.

24. El Kady N, Khedr H, Yosry M and El Mekawi S: Perioperative assessment of coagulation in paediatric neurosurgical patients using thromboelastography. Eur J Anaesthesiol 26: 293-297, 2009.

25. Schietroma M, Carlei F, Mownah A, et al: Changes in the blood coagulation, fibrinolysis, and cytokine profile during laparoscopic and open cholecystectomy. Surg Endosc 18: 1090-1096, 2004.

26. Goobie SM, Soriano SG, Zurakowski D, McGowan FX and Rockoff MA: Hemostatic changes in pediatric neurosurgical patients as evaluated by thrombelastograph. Anesth Analg 93: 887-892, 2001.

27. Lison S, Weiss G, Spannagl M and Heindl B: Postoperative changes in procoagulant factors after major surgery. Blood Coagul Fibrinolysis 22: 190-196, 2011.

28. Sadler JE: von Willebrand factor assembly and secretion. J Thromb Haemost 7 Suppl 1: 24-27, 2009.

29. Geerts WH, Pineo GF, Heit JA, et al: Prevention of venous thromboembolism: the Seventh ACCP conference on antithrombotic and thrombolytic therapy. Chest 126: 338S-400S, 2004.

30. Zhao YM, Jiang M, Ji SD, et al: Anti-human VWF monoclonal antibody SZ-123 prevents arterial thrombus formation by inhibiting VWF-collagen and VWF-platelet interactions in Rhesus monkeys. Biochem Pharmacol 85: 945-953, 2013. 